\title{
Numerical investigation of a Hall thruster plasma
}

\author{
Subrata Roy and B. P. Pandey \\ Computational Plasma Dynamics Laboratory, Kettering University, 1700 West Third Avenue, \\ Flint, Michigan 48504
}

(Received 3 January 2002; accepted 12 June 2002)

\begin{abstract}
The dynamics of the Hall thruster is investigated numerically in the framework of a one-dimensional, multifluid macroscopic description of a partially ionized xenon plasma using finite element formulation. The model includes neutral dynamics, inelastic processes, and plasma-wall interaction. Owing to disparate temporal scales, ions and neutrals have been described by set of time-dependent equations, while electrons are considered in steady state. Based on the experimental observations, a third order polynomial in electron temperature is used to calculate ionization rate. The results show that in the acceleration channel the increase in the ion number density is related to the decrease in the neutral number density. The electron and ion velocity profiles are consistent with the imposed electric field. The electron temperature remains uniform for nearly two-thirds of the channel; then sharply increases to a peak before dropping slightly at the exit. This is consistent with the predicted electron gyration velocity distribution. () 2002 American Institute of Physics.
\end{abstract} [DOI: $10.1063 / 1.1498261]$

\section{INTRODUCTION}

The stationary plasma thruster is a class of closed drift (Hall) thrusters that have evolved in several countries as an attractive electrostatic propulsion device for orbit maneuvering and station keeping. The reason for such popularity is due to its superior thrust $\left(\sim 10^{2}-10^{3} \mathrm{mN}\right)$ and high efficiency $(>50 \%)$ over a wide range of currents. The acceleration in a Hall thruster is not inhibited by the space charge field in quasineutral plasma. ${ }^{1,2}$ In general, the electric propulsion devices allow for lower propellant mass by generating higher exhaust velocities than is otherwise possible with chemical rockets. However, the electric propulsion devices are more challenging in comparison with chemical propulsion devices; not only is obtaining the test results under real flight conditions difficult, but also the interaction of the plasma plumes with the spacecraft makes the problem highly nonlinear.

Hall thruster experimentation started in the early 1960s and due to the diligent Russian effort became an enabling technology for on-board propulsion in many low earth orbit (LEO) and geosynchronous equatorial orbit (GEO) satellites. ${ }^{3}$ Present day kilowatt level Hall thrusters offer specific impulses ranging from 1600 to $2000 \mathrm{~s}$ with $80 \mathrm{mN}$ to $\sim 1 \mathrm{~N}$ thrust. Increasing the efficiency of the Hall thruster is a challenge. While having a lifetime of close to $8000 \mathrm{~h}$, the choice of thruster size requires an optimum selection between efficiency and lifetime. ${ }^{4}$ The physics inside the Hall thruster has to be reasonably well understood in order to make any significant change in efficiency without compromising the lifetime. In addition, sputter yield prediction is of considerable interest to the electric propulsion community as it is intimately related to the lifetime issues. Despite ample theoretical and experimental efforts published in the literature, a recent study ${ }^{1}$ recognizes the need for an acceptable computational model that captures the details of electrons and plasma dynamics inside the thruster annular cavity.

Figure 1 shows the annular schematic of a stationary plasma Hall thruster with an external hollow cathode. The propellant xenon $(\mathrm{Xe})$ gas is injected near the anode at the rear end. Concentric dielectric walls bound the acceleration channel. Hall thrusters are characterized by comparatively long acceleration channel $(0.02-0.03 \mathrm{~m})$ and a dominant radial magnetic field $(\sim 200 \mathrm{G})$ concentrated about the channel exit. The presence of such a strong radial magnetic field inhibits the transverse motion of the electrons, since their negligible gyration radius in comparison with the width of the channel virtually glue them to the radial field lines. The resulting high impedance of the electrons in the axial direction helps to maintain an electric field between anode and cathode. The ions, on the other hand, have a large gyration radius and therefore, will behave as if there is no radial magnetic field. This will result in ions streaming out of the device, accelerating down the potential like unmagnetized plasma.

Hall thruster dynamics is quite complex and thus, certain simplifying assumptions are necessary before attacking the problem in totality. Recently, several authors have carried out numerical studies of Hall thrusters in the framework of hybrid, as well as fluid, models. ${ }^{5-13}$ In the hybrid particle-incell (PIC) model ions and neutrals are treated as particles, while electrons are streaming as fluid. ${ }^{9,10}$ In the fluid formulation ${ }^{5,71-13}$ all species are described by their respective macroscopic equations. Several one- and twodimensional models are available in the literature. Manzella, ${ }^{5}$ Boeuf and Garrigues, ${ }^{6}$ Ahedo et $a .^{7}{ }^{7}$ (to name a few with no particular order) have documented one-dimensional (1D) Hall thruster simulations. Fife ${ }^{10}$ (and references therein), Keidar et al., ${ }^{12}$ and Roy and Pandey ${ }^{13}$ document the two dimensional (2D) numerical results. These studies aim towards predicting high fidelity solution details inside the thruster while simulating real flight conditions, and towards better 


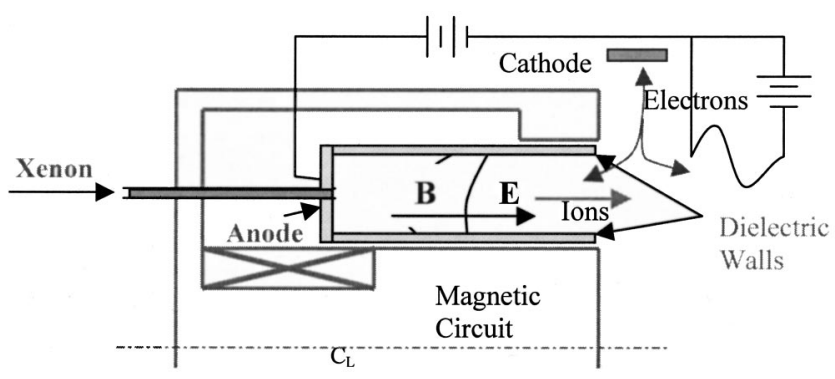

FIG. 1. Hall thruster schematic shows partially ionized gas flow inside the channel under the influence of electric and magnetic fields.

prediction of the performance and design issues. Despite some advances in our understanding of the thruster plasma dynamics, the need for further investigation of the subject to improve and optimize the thruster design remains.

As a complement to our previous work, ${ }^{13}$ the present study generalizes the model to partially ionized plasma incorporating the neutral dynamics and the effect of inelastic processes. Here a 1D numerical model is employed to study the physics of the acceleration process inside the channel with plasma-wall interaction. It is anticipated that the result will provide the basic insight of the underlying thruster physics, namely, the inter-relationship between the plasma temperature and ion and neutral densities, the ion and electron velocities and currents. To the best of our knowledge, no such study is available in the literature.

\section{PHYSICAL PROCESSES IN A THRUSTER PLASMA}

The Hall thruster plasma is partially ionized gas, consisting of electrons, ions, and neutral particles. The neutral particle is xenon, which is supplied externally through the inlet/ anode. The plasma is formed primarily through the collisional ionization via the electron impact to the incoming neutral propellant, downstream of the channel, in a narrow region. The plasma in the thruster is assumed to be quasineutral, i.e., electron number density $n_{e}$ is locally equal to ion number density $n_{i}$. The assumption of quasineutrality is valid except in the thin sheath layer near the walls. Sheath dynamics is not considered in the present work. The plasma in the thruster is sustained within the annular discharge chamber by an axial electric field $E_{Z}$ established between the external cathode and the anode located at the inlet. The electrons coming out of the external cathode flow towards the anode across the radial magnetic field established by the electromagnets. The interaction of these electrons with the crossed axial electric field $E_{Z}$ and radial magnetic field $B_{r}$ redirects the electron in the azimuthal direction, greatly reducing the electron conductivity in the axial direction. As an interesting consequence, despite the plasma being quasineutral, over the channel width the electrostatic field is maintained due to the charge separation inside the acceleration channel.

In such partially ionized plasma, several important elastic and inelastic processes can take place simultaneously. The elastic collision involves only the exchange of momentum and energy between colliding particles, whereas inelastic processes like ionization, recombination, charge-exchange collision, plasma-wall interaction, secondary emission, sputtering, etc. can be responsible for redistributing the number density of the particles along with its momentum and energy. As described in Appendix A, not all processes are equally probable. For example, the momentum exchange due to Coulomb interaction is not as important as the plasma-neutral momentum exchange. Furthermore, plasma-wall interaction may play an equally important role as the plasma-neutral collision in axial electron transport to the anode.

\section{GOVERNING EQUATIONS}

Owing to small inertia, electron response time is much faster than the ion response time. As a result, electrons will attain steady state much faster than ions. Keeping this in mind, electron momentum and energy equations are solved as steady state equations, whereas for ions and neutrals, a set of time independent continuity and momentum equations are simultaneously solved. The axisymmetric cylindrical thruster plasma is modeled by 1D geometry, where $z$ corresponds to axial direction and $\theta$ is along the azimuth. The following one-dimensional equations are solved in the present work.

Electron momentum equation:

$$
\begin{aligned}
V_{e z} \frac{\partial V_{e z}}{\partial z}= & -\frac{1}{m_{e} n_{e}} \frac{\partial}{\partial z}\left(p_{e}\right)-\frac{e}{m_{e}} E_{z} \\
& -\left(\frac{\omega_{c}^{2}}{\nu_{e i}+\nu_{e n}+\alpha_{B} \omega_{c}}\right) V_{e z} \\
& -\nu_{e i}\left(V_{e z}-V_{i z}\right)-\nu_{e n}\left(V_{e z}-V_{n z}\right) \\
& -\left(\frac{S}{n_{e}}\right)\left(V_{e z}-V_{n z}\right)+\nu_{w} V_{e z},
\end{aligned}
$$

where $m_{e}$ is the electron mass, $n_{e}$ is the electron number density. $V_{e z}, V_{i z}, V_{n z}$ are, respectively, electron, ion, and neutral axial velocities. $V_{\theta}=E_{Z} / B_{r}$ is the azimuthal electron drift velocity, $p_{e}=n_{e} T_{e}$ is the electron pressure with $T_{e}$ as the electron temperature in $\mathrm{eV}, E_{z}$ is the axial electric field, $\omega_{c}=e B / m_{e}$ is the electron-cyclotron frequency, and the source term due to ionization, recombination, and charge exchange is $S=S_{\text {recomb }}+S_{\text {ioniz }}+S_{\text {cex }}$. The electron-ion $\left(\nu_{e i}\right)$, electron-neutral $\left(\nu_{e n}\right)$, and electron-wall $\left(\nu_{w}\right)$ collision frequencies are defined in Appendix A. The following relation between azimuthal and axial velocities is utilized:

$$
V_{\theta}=\left(\frac{\omega_{c}}{\nu_{e i}+\nu_{e n}+\alpha_{B} \omega_{c}}\right) V_{e z}=\Omega V_{e z},
$$

where, $\alpha_{B}$ is the Bohm diffusion coefficient and $\Omega$ is the Hall parameter. The typical value of the Hall parameter varies between 100-1000.

The dynamics of the electron is determined by the pressure gradient, by the electric and magnetic forces, and by collisional exchange of momentum in Eq. (1). In the regions of sharp flow gradients, the effect of the convective term may become finite and therefore, the convective term is retained in this formulation. Similarly, since collision time scales are much larger than the electron-cyclotron gyration time scale, one may ignore elastic and inelastic collision 
terms in comparison with the Lorentz force term $V \times B$ in the momentum equation. Such an approach will exclude the dynamics of the momentum exchange, as well as the effect of ionization and recombination, severely limiting the applicability of the model to the thruster plasma. Furthermore, in addition to the presence of electron-ion and electron-neutral collisions, the electron-wall collision is thought to play an important in the electron transport. ${ }^{1}$ Therefore, all the collision terms are retained in the electron momentum Eq. (1).

It is known that the classical short-range, binary collision between plasma particles $\nu_{e i}$ and plasma-neutrals $\nu_{e n}$ is not sufficient to explain the cross field transport of the electrons and such behavior is explained either by invoking Bohm diffusion ${ }^{7}$ or by invoking plasma side-wall interaction. ${ }^{1,6}$ We model the plasma-wall interaction by introducing the electron-wall collision frequency $\nu_{w}$ (please see Appendix A for details). Further, the effect of anomalous Bohm conductivity has been included qualitatively by including the equivalent frequency $\nu_{B}=\alpha_{B} \omega_{c}$, which incorporates the effect of magnetic field fluctuations.

Neglecting the effect of radiation, viscous dissipation, and thermal conduction, the electron energy equation can be written as

$$
\begin{aligned}
\frac{d}{d z}[ & \left.n_{e} V_{e z}\left\{\frac{m_{e}\left(1+\Omega^{2}\right) V_{e z}^{2}}{2}+\frac{5}{2} T_{e}\right\}\right]-n_{e} e V_{e z} \frac{d \varphi}{d z} \\
= & 3 \frac{m_{e}}{m_{i}} n_{e} \nu_{e i}\left(T_{i}-T_{e}\right)+3 \frac{m_{e}}{m_{n}} n_{e} \nu_{e n}\left(T_{n}-T_{e}\right) \\
& +S\left(\frac{3}{2} T_{e}+\alpha E_{i}\right)-n_{e} \nu_{w} E^{\prime} .
\end{aligned}
$$

Here $T_{e}, T_{i}(\sim 0.1 \mathrm{eV})$ and $T_{n}(\sim 0.1 \mathrm{eV})$ are electron, ion, and neutral temperatures in $\mathrm{eV}$, respectively, and $E_{i}$ is the ionization energy of the xenon. Further,

$$
\nu_{w} E^{\prime}= \begin{cases}\frac{2 T_{e} V_{\mathrm{the}}}{h} e^{\varphi}\left[(2-\varphi)-\delta\left(2 \frac{T_{\mathrm{se}}}{T_{e}}-\varphi^{\prime}\right)\right] ; & \varphi^{\prime} \leqslant 0, \\ \frac{4 T_{e} V_{\text {the }}}{h}\left(1-\frac{T_{\mathrm{se}}}{T_{e}}\right) ; & \varphi \geqslant 0 .\end{cases}
$$

Here, $T_{\mathrm{se}}$ is the temperature of secondary electrons. Equation (3) includes the effect of Joule heating, the contribution due to the exchange of random thermal energy and due to the ionization and recombination and interaction of the plasma with the wall. The convective flux of kinetic energy includes the flux of azimuthal electron kinetic energy $V^{2}=V_{e z}^{2}$ $+V_{e \theta}^{2}$. The value of $\alpha$ is between (2-3). ${ }^{7}$

The ion continuity equation is

$$
\frac{\partial n_{i}}{\partial t}+\frac{\partial\left(n_{i} V_{i z}\right)}{\partial z}=S-\nu_{w} n_{i}
$$

In an ion momentum, the momentum exchange due to collision with electrons will not be significant, as the ion mean free path is generally larger $(\sim 0.3 \mathrm{~m})$ than the size of the thruster $(\sim 0.02 \mathrm{~m})$. Also, we consider ions as unmagnetized, since the gyration radius of ions is typically large for a $200 \mathrm{G}$ field with an ion velocity $4 \times 10^{3} \mathrm{~m} / \mathrm{s}$. Thus we ignore

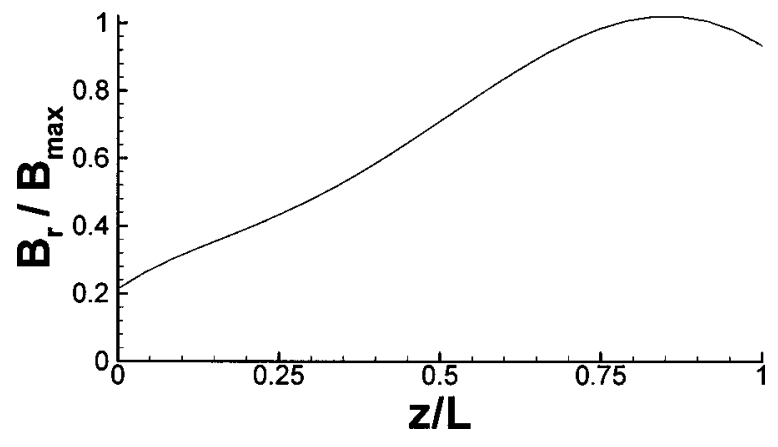

FIG. 2. Imposed magnetic field distribution. The magnetic field has a maximum near the exit plane.

the effect of magnetic field on the ion transport. The pressure term in the ion momentum equation can be ignored, as the thermal energy of the ions is much smaller than their kinetic energy, i.e., $T_{i} \ll m_{i} V_{i}^{2}$. Then the ion momentum becomes

$$
\begin{aligned}
\frac{\partial V_{i z}}{\partial t}+V_{i z} \frac{\partial V_{i z}}{\partial z}= & \left(\frac{e}{m_{i}}\right) E_{z}+\left(\frac{m_{e}}{m_{i}}\right) \nu_{e i}\left(V_{e z}-V_{i z}\right) \\
& -\left(\frac{m_{n}}{m_{i}}\right) \nu_{i n}\left(V_{i z}-V_{n z}\right) \\
& -\left(\frac{S}{n_{e}}\right)\left(V_{i z}-V_{n z}\right)+\nu_{w} V_{i z} .
\end{aligned}
$$

Neutral continuity,

$$
\frac{\partial n_{n}}{\partial t}+\frac{\partial\left(n_{n} V_{n_{z}}\right)}{\partial z}=-S_{n} .
$$

Here $S_{n}=S_{\text {recomb }}+S_{n, \text { ioniz }}+S_{\text {cex }}$ and $S_{n, \text { ioniz }}=k_{i}^{0+} n_{e} n_{n}$ $+k_{i}^{0++} n_{e} n_{n}$. Equations (1)-(6) are supplemented with the current and mass conservation equations, respectively, as

$$
\begin{aligned}
& e n_{i}\left(V_{i z}-V_{e z}\right)=J_{T}, \\
& m_{n} n_{n} V_{n z}+m_{i} n_{i} V_{i z}=\frac{\dot{m}}{A} .
\end{aligned}
$$

Here $J_{T}=I_{d} / A$ is the total current density, $I_{d}$ is the total discharge current, $A$ is the cross section of the thruster channel, and $\dot{m}$ is the mass flow rate.

Before numerically solving the above set of basic equations, the physical variables are normalized using experimental data. The mass flow rate of the propellant is $\dot{m}=\rho V A$. Then the flux of the propellant is $\Gamma=10^{23} \mathrm{~m}^{-2} \mathrm{~s}^{-1}$. Temperature $T_{e}$ is normalized to the first ionization potential of xenon, $T_{*}=E_{i}(12.1 \mathrm{eV})$. Then all dependent variables can be normalized from $V_{*}=\sqrt{ }\left(T_{*} / m_{i}\right) \mathrm{m} / \mathrm{s}, \quad n_{*}$ $=\Gamma_{*} / V_{*} \mathrm{~m}^{-3}, \nu_{*}=\sigma_{*} \Gamma_{*} \mathrm{~s}^{-1}$, where $\sigma_{*}=\sigma_{0} \sqrt{ }\left(m_{i} / m_{e}\right)$, $\sigma_{0} \cong 3.6 \times 10^{-20} \mathrm{~m}^{2}$ for Xe (see Appendix A for details). The fundamental length scale can be defined in terms of the characteristic velocity and collisional frequency as, $l_{0}$ $=V_{*} / \nu_{*}$. The time scale is $t_{0}=\nu_{*}^{-1}$.

Initial and boundary conditions: In order to numerically solve the formulations (1)-(8), proper initial and boundary condition specifications are necessary to make the problem well posed. In a typical Hall thruster experiment, the radial field is dominant in comparison with the axial field. Thus, a 


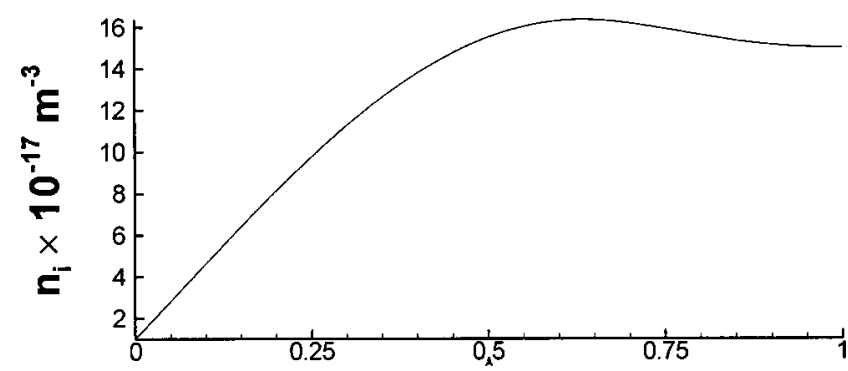

FIG. 3. Ion density increases towards the exit by an order of magnitude.

one-dimensional radial magnetic field is considered in the present work. A shifted Gaussian (bell-shaped) magnetic field profile is assumed (see Fig. 2), which reaches maximum just upstream of the exit plane,

$$
B_{r}(z)=B_{0}(z)+B_{\max } \exp \left(-\left(z-z_{\text {exit }}\right)^{2}\right) .
$$

The neutral number density at the inlet is assumed as given and is equal to the reference density. The axial ion velocity is not fixed at the inlet. Under typical conditions, next to the anode, a plasma sheath (typical width $\sim$ Debye length) forms and ions must flow into the sheath from the quasineutral region. ${ }^{14}$ The axial velocity is near zero close to the anode and then begins to rise at the edge of the acceleration zone and reaches maximum velocity beyond the exit. ${ }^{15}$ Such flow behavior has also been observed in the classical nozzle problem, where flow changes smoothly from subsonic (in the narrow region) to the supersonic flow in the divergent region. Therefore, a sonic point, where the flow velocity equals the characteristic speed of the medium, is always expected at the exit. In conformity with the available experimental results and numerical model ${ }^{7}$ we shall impose ion velocity at the exit boundary, whereas electron velocity is assumed zero at the inlet. At the inlet, the plasma density is fixed $n_{i}$ $=0.14 n_{*}$ and, a homogeneous Neumann condition for the electrostatic potential is imposed. At the downstream boundary (thruster exit plane), we specify an electron temperature $T_{e}=10 \mathrm{eV}$, that is close to the experimental results. ${ }^{16}$

Since at the cathode, the potential is zero, a vanishing potential is assumed at the outlet. For neutral and ion densities along with the electron velocity, a homogeneous Neumann condition is assumed at the exit. The electron temperature is fixed to $T_{e}=0.44 T_{*}$ at the outlet. The velocity of the neutral is consistently calculated from the mass flow equation.

The basic set of Eqs. (1)-(8) is solved using finite element based numerical model detail of which is given in Appendix B.

\section{RESULTS AND DISCUSSION}

Equation set (1)-(8) has been solved over a computational domain $(z / L: 0,1)$ where $L$ is the channel length with the exit plane located at $2 \mathrm{~cm}$. The mesh consists of 40 equal length 1D quadratic finite elements (i.e., 81 nodes) for all numerical results presented here.

The ion number density (Fig. 3) increases rapidly from a base value of $2.8 \times 10^{17} \mathrm{~m}^{-3}$ and attains a maximum value

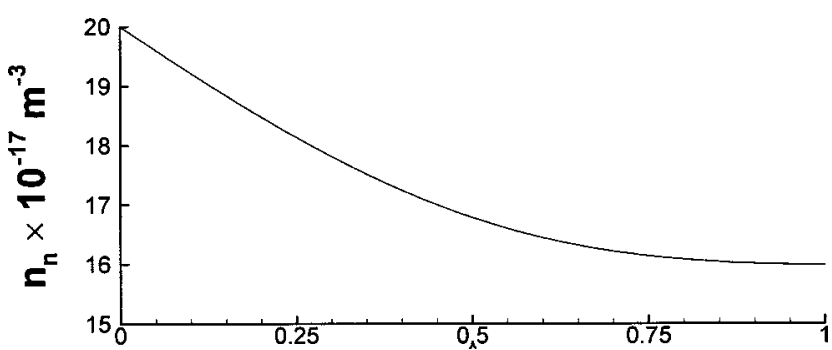

FIG. 4. The neutral density decreases toward the exit and reaches a plateau.

$1.6 \times 10^{18} \mathrm{~m}^{-3}$ upstream of the acceleration channel before decreasing near the exit. The experimental results ${ }^{14}$ show that the plasma density reaches its peak value inside the acceleration channel, at the right bottom corner of the exit plane. In this region, the radial magnetic field is maximum and thus a large number of electrons are inhibited from moving in the axial direction, resulting in the high probability of impact ionization and hence, plasma production. The maximum plasma density inside the acceleration channel is in agreement with the fact that the ionization channel is well inside the thruster. The computed ion number density profile suggests that the ionization region is well inside the channel, at about 0.60 .

The rapid increase in the ion number density is reflected in the rapid decrease in the neutral number density (Fig. 4) from $2 \times 10^{18} \mathrm{~m}^{-3}$ to approximately $1.6 \times 10^{17} \mathrm{~m}^{-3}$. This is consistent with the fact that as the neutral enters the thruster chamber it undergoes the impact ionization. Our results are at variance with the result of Bouef and Garigues ${ }^{6}$ where the minimum in neutral density is not reflected in the corresponding increase of the ion density. This has been attributed to the fact that once ionization takes place, ions due to the presence of electric field are accelerated and leave the acceleration channel faster than the replenishment of the neutral. A correlation between ion and neutral density is apparent in the present case. This correlation may be attributed to the temperature dependent, self-consistent calculation of the ionization rate.

Figure 5 describes the axial ion velocity profile. The velocity peaks downstream of the channel, before the exit. This indicates that the location of the acceleration channel is inside the acceleration channel at 0.75 . The ions are accelerated mainly due to the presence of the potential gradient, which is maximum near the channel exit, Fig. 6. Further, one may infer from the location of the acceleration channel that

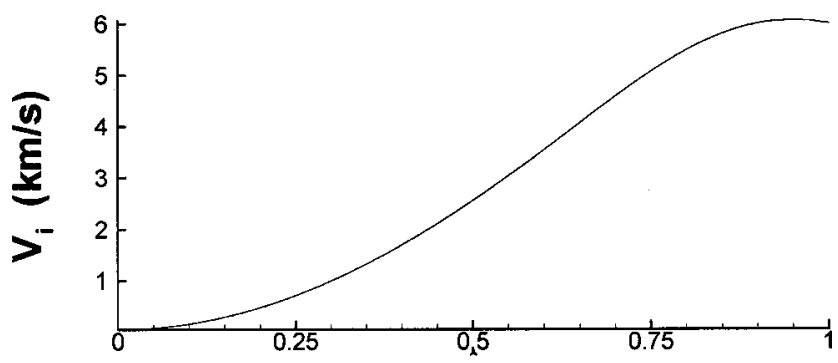

FIG. 5. The ion velocity profile suggests that the ions are accelerated towards the exit. 


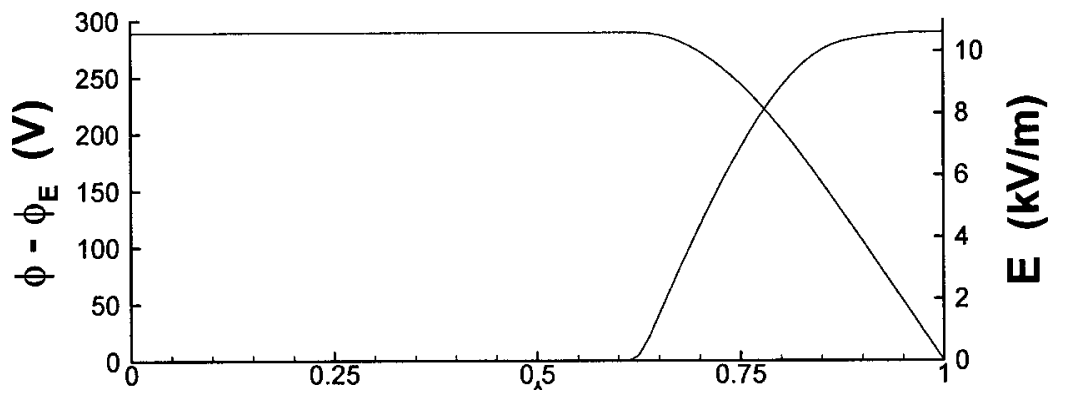

FIG. 6. Electric field $E$ and potential difference $\phi$ $-\phi_{E}$. The potential remains unchanged for $2 / 3$ rd of the channel and then sharply drops to the exit potential $\phi_{E}$.

the width of the ionization region is narrower $(\sim 0.15)$ than the width of the acceleration channel $(\sim 0.25)$. This is in conformity with the results of Bishaev and Kim. ${ }^{14}$

Figure 6 shows the potential profile inside the acceleration channel. The potential is highest at the inlet (near anode) and zero at the outlet (near cathode). We see that the potential has a zero gradient inside the thruster channel, similar to the experimental data. ${ }^{14}$ However, the computed potential vanishes at the channel exit, while observations ${ }^{14,17}$ indicate that only one-half to one-third of the potential drop takes place downstream of the thruster exit. This difference is due to the imposition of the zero potential boundary condition at the exit plane in numerical simulation, i.e., full potential drop is forced to occur inside the channel.

Figure 7(a) shows the electron velocity profile. This is consistent with the physical picture, where the electrons from the cathode, located just outside the chamber of a Hall thruster, is accelerated towards the anode. Large negative velocity near the exit is consistent with the large electric field (Fig. 6, dotted line), which are responsible for accelerating the electrons towards the inlet. These inward moving elec-

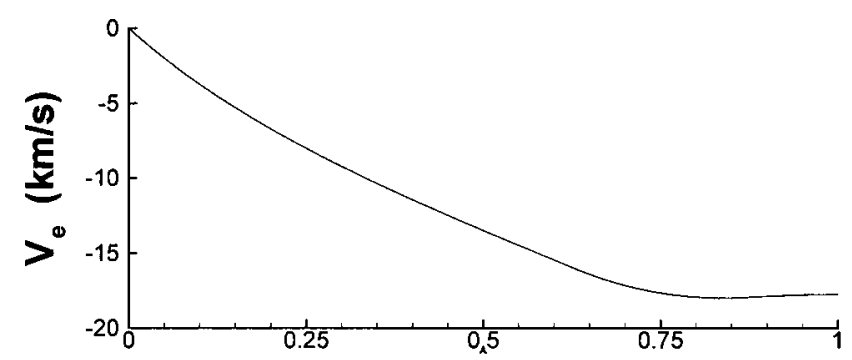

(a)

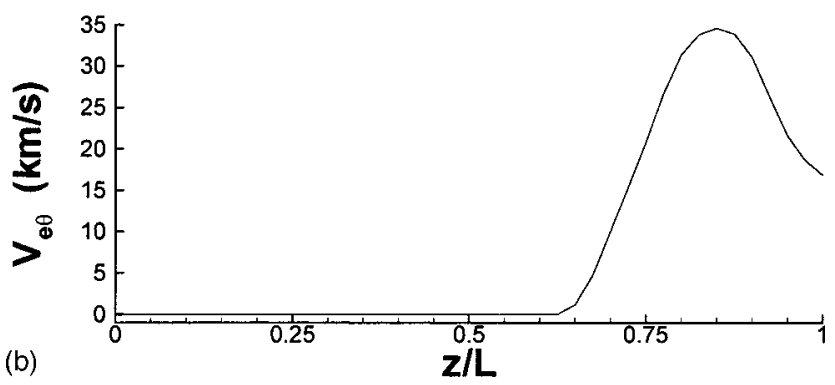

FIG. 7. (a) Electron velocity. Electrons are moving toward the anode (located at $z=0$ ). (b) Electron drift velocity is maximum just upstream of the channel exit. trons, on their way to the anode, collide with the neutrals and ionize them. As a result, electron velocity decreases towards the anode, as reflected in the figure.

The axial electron motion is shown in Fig. 7(b) The azimuthal electron drift velocity is a consequence of the crossed electric and magnetic field and gives rise to Hall current density, $J_{H} \approx e n_{e} V_{\theta}$. The peak in the azimuthal velocity downstream is consistent with the electron temperature profile (Fig. 8). The drift velocity may significantly enhance the ionization rate, at least by an order of magnitude, as drift speed becomes comparable to the electron thermal speed. ${ }^{15}$ One further notes that Hall current density may become several times larger than the discharge current and may give rise to a self-field, which can play an important role in controlling performance of the thruster.

Figure 8 describes the electron temperature profile. The increase in the temperature is not uniform in the channel. The maximum increase occurs just downstream of the center of the channel. This peak in electron temperature can be attributed to the Ohmic heating due to the maximum gyration energy in this region. This trend in temperature distribution is similar to the results reported in the literature. ${ }^{7}$ The numerical prediction also resembles the measured electron temperature near the exit. ${ }^{14,15,18}$ The uncertain nature of temperature data inside the channel was noted by Bishaev and Kim. ${ }^{14}$ However, for a $3 \mathrm{~kW}$ class thruster, ${ }^{18}$ the experimental electron temperature peak is spread like a radial line concentrated near the channel exit. Our 1D numerical electron temperature results do not exactly reproduce this profile pointing to the limitation of the present 1D model (Fig. 9).

\section{CONCLUSIONS}

In this paper, a finite element, 1D formulation of partially ionized plasma using the multicomponent fluid equa-

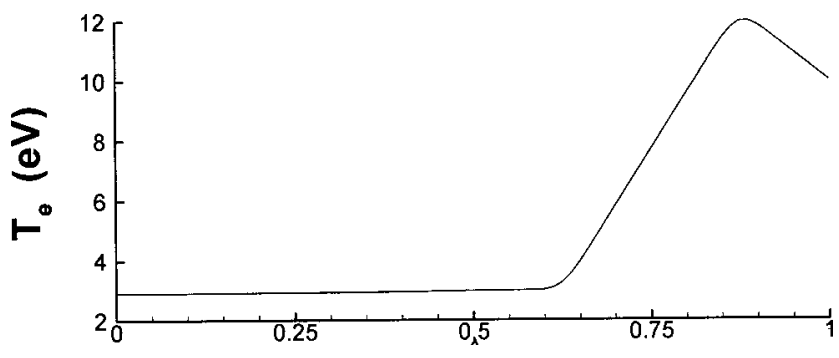

FIG. 8. Electron temperature in eV. It can be seen from the curve that, near the exit plane, the computed profile is in agreement with the experiment (Refs. 15, 20). 


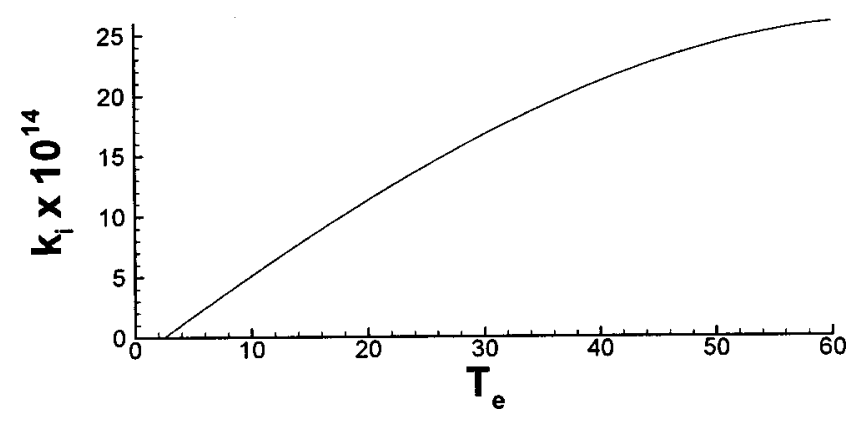

FIG. 9. Ionization rate $k_{i}$ as a function of electron temperature in $\mathrm{eV}$ is plotted as the sum of all the ionization rates $k_{i}=k_{i}^{0+}+k_{i}^{0++}+k_{i}^{1++}$ corresponding to $\mathrm{Xe} \rightarrow \mathrm{Xe}^{+}, \mathrm{Xe} \rightarrow \mathrm{Xe}^{++}$, and $\mathrm{Xe}^{+} \rightarrow \mathrm{Xe}^{++}$, respectively.

tion is given and the model is applied to study the dynamics of the Hall thruster. Owing to the disparate temporal scales, the ions have been described by the set of time-dependent equations, whereas electrons have been described by the steady state equations. Based on the experimental data for $\mathrm{Xe} \rightarrow \mathrm{Xe}^{+}, \mathrm{Xe} \rightarrow \mathrm{Xe}^{++}$, and $\mathrm{Xe}^{+} \rightarrow \mathrm{Xe}^{++}$ionization processes, a third order polynomial has been used in electron temperature as a fit to these processes. Such a polynomial has been used for self-consistent calculation of the ionization rate in the ion continuity equation. For the neutral continuity equation, a third order polynomial corresponding to $\mathrm{Xe}$ $\rightarrow \mathrm{Xe}^{+}$and $\mathrm{Xe} \rightarrow \mathrm{Xe}^{++}$has been used.

The plasma and neutral density profiles are in good agreement with reported experimental data. ${ }^{14,15}$ The selfconsistent calculation displays a direct correlation between the ion and neutral densities. The electron temperature predicts a maximum downstream of the channel exit and is in agreement with the experimental observations ${ }^{18}$ that show a peak next to the exit for a $3 \mathrm{~kW}$ class thruster. The potential profile agrees with the recent experimental studies. ${ }^{15}$ The axial ion velocity distribution shows that ions are accelerated down the channel, as would be expected for a thruster plasma.

Our 1D model has several simplifying assumptions, which will be relaxed in subsequent work. Namely, the quasineutrality assumption is not valid near the anode, and the sheath effect should be taken into consideration. The issue of anomalous plasma transport near the channel exit is crucial in understanding the dynamics and future work will include this phenomena by modeling plasma sidewall interactions, in a proper, 2D framework. This calls for generalization of $1 \mathrm{D}$ model to $2 \mathrm{D}$, which shall be carried out to bring the geometry and physics of the problem close to the real thruster dynamics. Furthermore, a time-dependent model is necessary for a self-consistent study of plasma-wall interactions and anomalous electron transport, which may be caused by the presence of very low-frequency oscillation in the system.

\section{ACKNOWLEDGMENTS}

The authors gratefully acknowledge the technical discussions with the electric propulsion group of Glenn Research Center.
This work is supported by NASA Research Grant No. NAG3-2520 with David Jacobson as the technical monitor.

\section{APPENDIX A: CHARGE INTERACTION PROCESS IN A THRUSTER}

The main force responsible for charged particle selfinteractions, as well as with each other, is the long range Coulomb force. Because of the long-range nature of the Coulomb force, plasma particles can be deflected over the Debye length $\lambda_{D}$. The electron-ion collision frequency is

$$
\nu_{e i}=\frac{4 \sqrt{2 \pi}}{3 \sqrt{m_{e}}} \frac{e^{4} n_{i} L_{e}}{T_{e}^{3 / 2}}=\frac{L_{e}}{3 \sqrt{2 \pi}}\left(\frac{n_{i}}{n_{e}}\right)\left(\frac{\omega_{p e}}{n_{e} \lambda_{D e}^{3}}\right) .
$$

Here, $\omega_{p e}^{2}=4 \pi n_{e} e^{2} / m_{e}$ is the square of the electron plasma frequency with an electron mass $m_{e}$ and charge $e, \lambda_{D e}^{2}$ $=T_{e} /\left(4 \pi n_{e} e^{2}\right) . L_{e}=\ln (\Lambda)$ is the Coulomb logarithm. It has a typical value around 10-20. The collision term determined by electron-electron and ion-ion collision is equal to

$$
\nu_{\alpha \alpha}=\frac{4 \sqrt{2 \pi}}{3 \sqrt{m_{\alpha}}} \frac{e^{4} n_{\alpha} L_{\alpha}}{T_{\alpha}^{3 / 2}}=\frac{L_{\alpha}}{3 \sqrt{2 \pi}}\left(\frac{\omega_{p \alpha}}{n_{\alpha} \lambda_{D \alpha}^{3}}\right) .
$$

The ratio between different collision frequencies is

$$
\frac{\nu_{e e}}{\nu_{e i}} \approx \sqrt{2} ; \frac{\nu_{i i}}{\nu_{e i}} \approx \sqrt{2}\left(\frac{m_{e}}{m_{i}}\right)^{1 / 2} \frac{L_{i}}{L_{e}}
$$

for $n_{e} \approx n_{i}$ and $T_{i} \approx T_{e}$. The momentum exchange terms due to electron-electron and ion-ion interaction will not be important in comparison with the electron-ion momentum exchange as the relative drift between similar particles is small in comparison with the drift between electrons and ions. Therefore, $\nu_{e e}, \nu_{i i}$ frequencies shall be ignored from further consideration.

For typical conditions of a Hall thruster, the effect of Coulomb collision $\left(\nu_{e i}\right)$ may not be significant in comparison with the plasma-neutral collision $\nu_{e n} .{ }^{6}$ Further, electron-wall collisions may play an equally important role as the plasma-neutral collision in momentum transport. Plasma interaction with the walls leads to recombination and secondary emission, thermal losses, and electron diffusion. ${ }^{7}$ The probability of recombination is considerable at low electron energy as the electron-ion interaction time is sufficiently large. The following recombination process may occur: ${ }^{19}$

$$
\begin{aligned}
& e+\mathrm{Xe}^{+}+e \rightarrow \mathrm{Xe}+e, \quad e+\mathrm{Xe}^{+}+\mathrm{Xe}^{+} \rightarrow \mathrm{Xe}+\mathrm{Xe}^{+}, \\
& e+\mathrm{Xe}^{+}+\mathrm{Xe} \rightarrow \mathrm{Xe}+\mathrm{Xe}, \quad e+\mathrm{Xe}^{+} \rightarrow \mathrm{Xe}+h \nu .
\end{aligned}
$$

Not all processes are equally probable. For example, the probability of the recombination with the ion as a third body (second reaction) is always negligibly small compared to the first reaction.

The recombination in the presence of a neutral body is important at the low degree of ionization. The recombination 
coefficient $\alpha$ can be approximated as $^{20} \quad \alpha=1.09$ $\times 10^{-20} n_{e} T^{-9 / 2} \mathrm{~m}^{3} / \mathrm{s}$. Then the recombination rate can be written as

$$
S_{\text {recom }}=-1.09 \times 10^{-20} T^{-9 / 2} n_{e}^{3} \mathrm{~m}^{3} / \mathrm{s},
$$

where assuming quasineutrality $n_{i}$ has been replaced by $n_{e}$.

The inelastic electron collision with the wall allows the electrons to move across the magnetic field toward the anode, giving rise to "near wall conductivity." Thus, for modeling the near wall conductivity, one needs to specify the secondary emission and sheath potential. Furthermore, it is believed that the wall with high secondary electron emission $\delta$ gives rise to high cross-field conductivity, since a large fraction of the incident energetic electrons are returned to the plasma as cold electrons with new guiding center drift along the direction of the electric field. Thus for a near wall sheath potential,

$$
\varphi^{\prime}=-\left[0.5+\ln \left\{(1-\delta)\left(\frac{m_{i}}{2 \pi m_{e}}\right)^{1 / 2}\right\}\right]
$$

electron-wall collision frequency, for a channel of width $h$ can be given as

$$
\nu_{w}=\left\{\begin{array}{l}
\frac{2 V_{\text {the }}}{h} e^{\varphi^{\prime}}(1-\delta) ; \quad \varphi^{\prime} \leqslant 0 \\
\frac{2 V_{\text {the }}}{h} ; \quad \varphi^{\prime} \geqslant 0
\end{array}\right.
$$

Here $\varphi^{\prime}=e \varphi^{\prime} / T_{e}$ and coefficient of secondary emission and for Boron nitride wall is given as

$$
\delta=0.198 \times T_{e}^{0.576} .
$$

Electron collisions with the xenon atom is the main source of ion production in propulsion plasma. The rate of ion production in plasma is determined by the total cross section of the process. Thus,

$$
S_{\text {ioniz }}=n_{e} n_{n}\left\langle V \sigma^{i}(V)\right\rangle=k_{e n}^{i} n_{e} n_{n},
$$

where, for the process constant $k^{i}=\left\langle V \sigma^{i}(V)\right\rangle$, the averaging is done over the velocities of the electrons whose energy is sufficient for ionization $m V^{2} / 2>E_{i}$. The ionization process can be described as $e+\mathrm{Xe} \rightarrow \mathrm{Xe}^{+}+e+e^{\prime}$, where $e$ and $e^{\prime}$ have different energy level. Processes like $\mathrm{Xe} \rightarrow \mathrm{Xe}^{++}$and $\mathrm{Xe}^{+} \rightarrow \mathrm{Xe}^{++}$may also play important role. The ionization source term, which takes into account all the above processes, is

$$
S_{\text {ionization }}=k_{i}^{0+} n_{e} n_{n}+k_{i}^{0++} n_{e} n_{n}+k_{i}^{1++} n_{e}^{2},
$$

where $0+, 0++$ represents the transition from neutral to singly and doubly ionized state, respectively, and $1++$ represents the transition from singly to doubly ionized state. The ionization cross section for Xe is given by Darwin, ${ }^{20}$

$\sigma^{j}(u)=2.66 \pi a_{0}^{2}\left(\frac{E_{i}^{H}}{E_{i}^{k \lambda}}\right)^{2} \xi_{k \lambda} \beta_{1}\left(\frac{u-1}{u^{2}}\right) \ln \left(1.25 \beta_{2} u\right)$.

Here $E_{i}^{H}$ is the ionization energy of hydrogen $(13.6 \mathrm{eV}), E_{i}^{k \lambda}$ is the threshold ionization energy, $\xi^{k \lambda}$ is the number of equivalent electrons in the $k$ th level, i.e., those electrons having principal and azimuthal quantum number same, $u$ $=E / E_{i}^{k \lambda}$, and $\beta_{1}$ and $\beta_{2}$ are adjustable parameters. For Xe gas $\beta_{1}=1.0$ and $\beta_{2}=0.87$. After doing the averaging over a Maxwellian electron, the process constant is given by ${ }^{10}$

$$
k_{e n}^{i}=4.13 \times 10^{-13} \frac{\int_{1}^{\infty} d u \ln \left(1.25 \beta_{2} u\right) e^{(-u / \theta)}\left(\frac{u-1}{u}\right)}{\theta^{3 / 2}},
$$

where $\theta=k T_{e} / E_{i}$. The process constant is typically about $1 \times 10^{-14}$ for $\mathrm{Xe} \rightarrow \mathrm{Xe}^{+}$. Processes like $\mathrm{Xe} \rightarrow \mathrm{Xe}^{++}$and $\mathrm{Xe}^{+} \rightarrow \mathrm{Xe}^{++}$may also play important role. For electron energies $<80 \mathrm{eV}, \sigma\left(\mathrm{Xe} \rightarrow \mathrm{Xe}^{++}\right)$can be calculated using Darwin's form with $\varepsilon^{i}=33.3 \mathrm{eV}$ and $\xi=3$.

No data exist for $\sigma\left(\mathrm{Xe}^{+} \rightarrow \mathrm{Xe}^{++}\right)$. Once again, Darwin's form can be used with $E_{i}=21.2 \mathrm{eV}, \xi=5$. The threshold energy $E_{i}=21.2 \mathrm{eV}$ is the difference between $E_{i}$ $\left(\mathrm{Xe}^{++}=33.3 \mathrm{eV}\right)$ and $E_{i}\left(\mathrm{Xe}^{+}=12.1 \mathrm{eV}\right)$. For the Hall thruster, $n_{e} n_{n} / n_{e}^{2} \sim 10^{2}$, one may conclude that the contribution due to $\mathrm{Xe}^{+} \rightarrow \mathrm{Xe}^{++}$to the ionization will be small in comparison to the $\mathrm{Xe} \rightarrow \mathrm{Xe}^{++}$since $k_{i}^{1++}<k_{i}^{0++}$.

A general third order temperature dependant polynomial can be fitted to the experimental value of ionization rate $k_{i}$ $=k_{i}^{0+}+k_{i}^{0++}+k_{i}^{1+}$. The matrix form is

$$
\left(\begin{array}{c}
k_{i}^{0+} \\
k_{i}^{0++} \\
k_{i}^{1++}
\end{array}\right)=\left(\begin{array}{cccc}
1.9435 \times 10^{-5} & -0.0068 & 0.6705 & -1.6329 \\
-3.0352 \times 10^{-5} & 0.0024 & 0.0515 & -0.1431 \\
-2.117 \times 10^{-5} & 0.0022 & -0.0119 & 0.0161
\end{array}\right)\left(\begin{array}{c}
T_{e}^{3} \\
T_{e}^{2} \\
T_{e}^{1} \\
T_{e}^{0}
\end{array}\right) \times 10^{-14}
$$

Figure 9 plots the sum of all three ionization rates as

$$
\begin{aligned}
k_{i}= & \left(-3.2087 \times 10^{-5} T_{e}^{3}-0.0022 T_{e}^{2}+0.7101 T_{e}-1.76\right) \\
& \times 10^{-14} .
\end{aligned}
$$

Please note that the above estimate of ionization rate is based on the Maxwellian distribution function. However, electrons are accelerated toward the anode by the imposed electric field before their head-on collision with the neutral gas particles. Therefore, a drifting Maxwellian will be an appropriate description of the electron distribution. It has been shown recently ${ }^{21}$ that for a drift speed between one to five times electron thermal velocity, the ionization rate increases by two to seven order of magnitude. Therefore, our ionization rate 
calculation may be slightly under estimated, especially near the thruster exit, where the electron drift may become comparable to the electron thermal speed.

Charge exchange is related to the transfer of one or more electrons between an atom and an ion. Slow propellant ions are created due to resonant charge-exchange collisions of the following type between the fast "beam" (current) ions and slow thermal neutrals,

$$
\begin{aligned}
& \mathrm{Xe}_{\text {fast }}^{+}+\mathrm{Xe}_{\text {slow }}^{0} \rightarrow \mathrm{Xe}_{\text {slow }}^{+}+\mathrm{Xe}_{\text {fast }}^{0}, \\
& \mathrm{Xe}_{\text {fast }}^{++}+\mathrm{Xe}_{\text {slow }}^{0} \rightarrow \mathrm{Xe}_{\text {slow }}^{++}+\mathrm{Xe}_{\text {fast }}^{0}, \\
& \mathrm{Xe}_{\text {fast }}^{++}+\mathrm{Xe}_{\text {slow }}^{0} \rightarrow \mathrm{Xe}_{\text {slow }}^{+}+\mathrm{Xe}_{\text {fast }}^{+} .
\end{aligned}
$$

The last process may not be significant in comparison with the preceding ones. The spatial volumetric production rate is given by $S_{\mathrm{CEX}}=n_{n} n_{i}\left\langle v_{i} \sigma\left(v_{i}\right)\right\rangle$, where relative collision velocity is taken to be the ion velocity. The process can be important for creating slow ions. The cross section for $\mathrm{Xe}-\mathrm{Xe}^{+}$for example, is given $\mathrm{by}^{22}$

$$
\sigma\left(\mathrm{Xe}-\mathrm{Xe}^{+}\right)=\left(142.21-23.30 \log _{10}(\Delta u)\right) \times 10^{-20} \mathrm{~m}^{2} .
$$

For a relative velocity between 10 and $2 \times 10^{3} \mathrm{~m} / \mathrm{s}$, the charge exchange cross section is between $10^{-20}-10^{-19} \mathrm{~m}^{2}$.

\section{APPENDIX B: FINITE ELEMENT BASED MODELING}

In the present work, a 1D finite element formulation is employed to solve Eqs. (1)-(8) which may be expressed as $L(\mathbf{U})=0$, where $\mathbf{U}=\left(n_{i}, n_{n}, V_{i}, V_{n}, V_{e}, T_{e}, \phi\right)$ and $L$ is a differential operator. The weak statement underlines the development of the range of numerical algorithms. Such an integral statement associated with (1)-(8) is

$$
\int_{\Omega} w L(\mathbf{U}) d \Omega=0,
$$

where $w$ denotes any admissible test function. ${ }^{23}$ Thereafter, the finite element (FE) spatial semidiscretization of the domain $\Omega$ of (1)-(8) employs the mesh $\Omega^{h}=\cup_{e} \Omega_{e}$ and $\Omega_{e}$ is the generic computational domain. Using superscript " $h$ " to denote "spatial discretization," the FE weak statement implementation for (B1) defines the approximation as

$$
u\left(x_{j}\right) \approx u^{h}\left(x_{j}\right)=\underset{e}{\cup} u_{e}\left(x_{j}\right) \text { and } u_{e}\left(x_{j}\right)=N_{k} \mathbf{U}_{e},
$$

where subscript $e$ denotes elements, and the trial space $\mathrm{FE}$ basis set $N_{k}\left(x_{j}\right)$ typically contains Chebyshev, Lagrange or Hermite interpolation polynomials complete to degree $k$, plus perhaps "bubble functions." 23 The spatially semidiscrete FE implementation of the weak statement $\mathrm{WS}^{h}$ for (B2) leads to

$$
\mathrm{WS}^{h}=S_{e}\left(\int_{\Omega_{e}} N_{k} L_{e}(\mathbf{U}) d \tau\right) .
$$

$S_{e}$ symbolizes the "assembly operator" carrying local (element) matrix coefficients into the global arrays. Application of the Green-Gauss divergence theorem in (B3) will yield natural homogenous Neumann boundary conditions and the surface integral that contains the unknown boundary fluxes wherever Dirichlet (fixed) boundary conditions are enforced.

Independent of the physical dimension of $\Omega$, and for general forms of the flux vectors, the semidiscretized weak statement of (B1) always yields an ordinary differential equation (ODE) system,

$$
\mathbf{M} d \mathbf{U} / d t+\mathbf{R}(\mathbf{U})=\mathbf{0}
$$

where $\mathbf{U}(t)$ is the time-dependent finite element nodal vector. The time derivative $d \mathbf{U} / d t$, is generally replaced by using a $\theta$-implicit or $\tau$-step Range-Kutta time integration procedure. In (B4), $\mathbf{M}=S_{e}\left(\mathbf{M}_{e}\right)$ is the "mass" matrix associated with element level interpolation, $\mathbf{R}$ carries the element convection information and the diffusion matrix resulting from genuine (not for Euler) or numerical elemental viscosity effects, and all known data. For the steady state, (B4) is usually solved using a Newton-Raphson scheme,

$$
\mathbf{U}_{\tau+1}^{i+1}=\mathbf{U}_{\tau+1}^{i}+\Delta \mathbf{U}^{i}=\mathbf{U}_{\tau}+\sum_{p=0}^{i} \mathbf{U}^{p+1},
$$

where

$$
\Delta \mathbf{U}^{i}=-[\mathbf{M}+\theta \Delta t(\partial \mathbf{R} / \partial \mathbf{U})]^{-1} \mathbf{R}(\mathbf{U})
$$

The obvious numerical issues will be associated with calculation of the "Jacobian" $\partial \mathbf{R} / \partial \mathbf{U}$ and inversion of the $\mathbf{M}$ $+\theta \Delta t(\partial \mathbf{R} / \partial \mathbf{U})$ matrix with sufficient accuracy. Here, an implicit $(\theta=1)$ time stepping procedure is employed.

The choice of time step is dictated by the CourantFredrich-Levy condition. ${ }^{24}$ The code uses variable time steps until the transient features die down as the iteration converges to a steady state. The solution is declared convergent when the maximum residual for each of the state variable becomes smaller than a chosen convergence criterion of $\in=10^{-4}$. Here, the convergence of a solution vector $\mathbf{U}$ on node $j$ is defined as the norm,

$$
\frac{\left\|\mathbf{U}_{j}-\mathbf{U}_{j-1}\right\|}{\left\|\mathbf{U}_{j}\right\|} \leqslant \epsilon .
$$

${ }^{1}$ A. I. Morozov and V. V. Savelyev, in Review of Plasma Physics, edited by B. B. Kadomtsev and V. D. Shafranov (Consultant Bureau, New York, 2000), Vol. 21, p. 203.

${ }^{2}$ V. V. Zurin, H. R. Kaufman, and R. S. Robinson, Plasma Sources Sci. Technol. 8, 1 (1999).

${ }^{3}$ R. Jankovsky, S. Tverdokhlebov, and D. Manzella, 35th Joint Propulsion Conference, 1999 (AIAA, Washington, D.C., 1999), AIAA-99-2949.

${ }^{4}$ V. Kim, J. Propul. Power 14, 736 (1998).

${ }^{5}$ D. Manzella, in Proceedings of the 24th International Electric Propulsion Conference, Moscow, Russia, 1995 (The Electric Rocket Propulsion Society, Worthington, OH, 1995), Vol. 1, p. 277.

${ }^{6}$ J. P. Boeuf and L. Garigues, J. Appl. Phys. 84, 3541 (1998).

${ }^{7}$ E. Ahedo, P. Martinez, and M. Martinez-Sanchez, Phys. Plasmas 8, 3058 (2001).

${ }^{8}$ K. Komurasaki and Y. Arakawa, J. Propul. Power 11, 1317 (1995).

${ }^{9}$ I. D. Boyd, L. Garrigues, J. Koo, and M. Keidar, 36th AIAA Joint Propul- 
sion Conference, Huntsville, AL, 2000 (AIAA, Washington, D.C., 2000), AIAA-2000-3520.

${ }^{10}$ J. M. Fife, Ph.D. thesis, MIT, 1998.

${ }^{11}$ A. Fruchtman, N. J. Fisch, and Y. Raitses, Phys. Plasmas 8, 1048 (2001).

${ }^{12}$ M. Keidar, I. D. Boyd, and I. I. Beilis, Phys. Plasmas 8, 5315 (2001).

${ }^{13}$ S. Roy and B. P. Pandey, in Proceedings of the 27th International Electric Propulsion Conference, Pasadena, California, 2001 (The Electric Rocket Propulsion Society, Worthington, OH, 2001), IEPC-2001-049.

${ }^{14}$ A. M. Bishaev and V. Kim, Sov. Phys. Tech. Phys. 23, 1055 (1978).

${ }^{15}$ W. A. Hargus, Jr. and M. A. Cappelli, J. Propul. Power 18, 159 (2002).

${ }^{16} \mathrm{~S}$. Kim, J. E. Foster, and A. D. Gallimore, 32nd AIAA Joint Propulsion Conference, Orlando, FL (AIAA, Washington, D.C., 1996), AIAA-962972, July 1996.

${ }^{17}$ J. M. Haas and A. D. Gallimore, Phys. Plasmas 8, 652 (2001)
${ }^{18}$ J. M. Haas and A. D. Gallimore, AIAA-00-3422, 36th Joint Propulsion Conference (JPC) Huntsville, AL, 16-19 July 2000.

${ }^{19}$ V. E. Golant, A. P. Zhilinsky, and I. E. Sakharov, Fundamentals of Plasma Physics (Wiley, New York, 1980), p. 67.

${ }^{20}$ M. Mitchner and C. H. Kruger, Partially Ionized Gases (WileyInterscience, New York, 1973).

${ }^{21}$ L. Conde, L. F. Ibanez, and C. Ferraro-Fontam, Phys. Rev. E 64, 046402 (2001)

${ }^{22}$ S. Pullins, Y. Chiu, D. Levandier, and R. Dressler, 38th Aerospace Sciences Meeting and Exhibit, Reno, NV, 2000 (AIAA, Washington, D.C., 2000), AIAA-2000-0603.

${ }^{23}$ S. Roy, Comput. Methods Appl. Mech. Eng. 184, 87 (2000).

${ }^{24}$ R. D. Richtmyer and K. W. Morton, Difference Methods for Initial-Value Problems, 2nd ed. (Interscience, Wiley, New York, 1967). 